\title{
1 Scavenging of Interleukin 6 Receptor by 2 Bioidentical Recombinant gp130 as \\ 3 Intervention in Covid-19 Exacerbation
}

$4 \quad$ Sebastian Lobentanzer ${ }^{1 *}$

$5 \quad{ }^{1}$ Institute of Pharmacology and Clinical Pharmacy, Goethe University, Frankfurt/Main, Germany.

6 Keywords

7 coronavirus; SARS; covid-19; gp130; interleukin 6; cytokine storm

\section{Abstract}

9 Acute exacerbations in the form of cytokine storm are the main cause of organ failure caused by

SARS-related coronaviruses and mediated by pro-inflammatory transcriptional programs in immune cells. While the effectors of these transcriptional changes have been sufficiently studied, there is a noticeable lack of interventional treatments for acute exacerbations. An important interface of innate immune signalling are the components of the JAK/STAT pathway, activated by interferons as well as pro-inflammatory cytokines such as interleukin (IL)-6. An intervention based on the IL-6 receptor antibody, tocilizumab, has already been proposed and is currently in clinical trial; however, the antibody does not differentiate between soluble and membrane-bound IL-6 receptors and thus may inhibit regenerative processes. Here, I propose utilising recombinant soluble protein gp130, also known as IL6ST, as a bioidentical scavenger molecule for activated 

soluble gp130 is a warranted alternative to monoclonal antibody therapy.

\section{Introduction}

23 Cytokine storm, with overproduction of pro-inflammatory cytokines and exaggerated response of

24 pro-inflammatory immune cell types in the blood, is the most common cause of acute respiratory distress syndrome and organ failure in severe viral infection (Bhaskar et al. 2020). Two major proinflammatory interleukins (ILs) are required for cytokine storm formation: IL-1 and IL-6 (Norelli et al. 2018). The acute-response cytokine IL-1 $\beta$ facilitates a sustained increase in IL-6, which subsequently activates the Janus kinase/signal transducer and activator of transcription (JAK/STAT) pathway through the gp130 receptor, thereby perpetuating inflammation (Tisoncik et al. 2012). Blocking this cascade upstream of JAK/STAT, for instance at the IL-6 receptor, may

31 be a valuable therapeutic option (Bhaskar et al. 2020).

32 Susceptibility to severe exacerbations of Covid-19 seems influenced by genetic disposition rather than simply by age; why some infections tend to present with severe clinical symptoms particularly in young, healthy adults is currently unknown (Tisoncik et al. 2012). To interfere with this exaggerated response, treatment with i.v. tocilizumab, the IL-6 receptor antibody, has been proposed and is currently in clinical testing. However, compared to approved indications for tocilizumab, such as rheumatoid arthritis (RA), the doses for i.v. application in acute hypercytokinaemia may have to be increased significantly, bearing the risk of severe adverse reactions and paradoxical immune stimulation (van Schie et al. 2015). Additionally, tocilizumab without distinction binds to soluble and membrane-bound IL-6 receptors alike, harbouring the risk 
41 for additional off-target adverse effects. For instance, the most common (very common, > 10\%)

42 adverse reaction to s.c. and i.v. tocilizumab is upper respiratory tract infection (Scott 2017). Serious

43 respiratory tract infections occurr in $8.5 \%$ of tocilizumab monotherapy patients in approved

44 indications, i.e., at low doses (Haraoui et al. 2017).

45 IL-6 as a neurokine conveys its effect on cells through the ubiquitously distributed gp130

46 transmembrane receptor protein, which is also known as IL-6 signal transducer (IL6ST),

47 immediately resulting in JAK/STAT activation. In most cases, IL-6 binds to the soluble IL-6 receptor (sIL6R) which then mediates gp130 homodimer activation on the cell surface (transsignalling). On the other hand, some cell types have been found to express a membrane-bound

50 form of the IL-6 receptor (mbIL6R): macrophages, neutrophils, some types of T-cells, and hepatocytes (Scheller et al. 2011). A multitude of studies has shown that the deleterious effects of IL-6 overexpression are caused by trans-signalling via the sIL6R, while the classic cis-signalling conveyed by mbIL6R is essential for anti-microbial effects and regeneration (Garbers et al. 2015).

54 Consequently, inhibition of IL-6 signalling via the IL6R antibody tocilizumab, the direct IL-6 antibody siltuximab, or small molecule TYK2-antagonists would inevitably interfere with the desired, classic IL-6 signalling through mbIL6R. In contrast, application of sIL6R-scavenging recombinant soluble gp130 protein (sgp130) could theoretically reduce detrimental trans-signalling while keeping classic signalling intact.

Whether these processes play a role in exacerbation of Covid-19 infection is unclear. To clarify human interleukin-related reaction to coronavirus exacerbation and feasibility of sIL6R scavenging

61 by sgp130 in these cases, I re-analysed expression data of a cohort of SARS-CoV patients, and 
62 assessed the tissue distribution of interleukin-related transcripts involved in their responses to the 63 infection.

\section{Results} patients and controls from the Toronto area in 2007 (Cameron et al. 2007) shows significant interleukin involvement in blood cell transcriptional processes surrounding exacerbation (Fig. 1). IL6R was up-regulated $(\sim 33 \%, \mathrm{p}=0.036)$ in patient blood pre-, but not post-exacerbation (indicated by arterial oxygen saturation nadir) compared to healthy controls, while IL-6 levels remained unchanged. The major pro-inflammatory cytokine IL-1 $\beta$ and its receptors were downregulated in patients pre- and post-exacerbation. In contrast, IL6R, IL17RE and IL22RA1 were up-regulated.

To get a better picture of IL6-related gene expression across relevant cell types, tissue-specific regulatory circuits of immune cells $(n=17)$ and control tissues $(n=16)$ were analysed, based on a dataset of transcriptional information from 808 human samples (Marbach et al. 2016). Since mbIL6R expression has been observed in immune and liver cells exclusively (Scheller et al. 2011), bronchial epithelial tissue was included as a control. Surprisingly, however, bronchial epithelia showed fourth-highest expression of the IL6R mRNA according to these data, more than liver cells, and surpassed only by monocytes and regulatory T cells (Supplementary Fig. 1). 
83 In a recent study performed on a humanised mouse model of hypercytokinaemia (utilising CAR Ttechnology), human monocyte-derived IL-1 and IL-6 were the drivers of cytokine release syndrome (Norelli et al. 2018). Conversely, differential expression in the Toronto cohort implies that hypercytokinaemia as a cause of clinical exacerbation of SARS-CoV may not be mediated by IL-1 $\beta$. IL-1 $\beta$ was decreased pre-exacerbation and did not increase above control levels even post exacerbation; IL-1 receptors were similarly down-regulated. Notably, most patients received glucocorticoids orally or intravenously, at a median of six days after symptom onset (DSO), which may have interfered with cytokine levels. Still, three pro-inflammatory cytokines and/or their receptors were elevated around the time of exacerbation (which occurred at a median of eight DSO): IL-6, IL-17, and IL-22. IL-17 and IL-22 have been described as relevant players in airway inflammation, and may control pro-inflammatory response as well as recovery parameters via complex interaction. However, their basal expression in the assessed tissues is extremely low, and their description in the literature is sparse.

IL-6, on the other hand, is a well-described pro-inflammatory cytokine whose receptors show high basal expression. IL6R expression was elevated in patients pre-exacerbation; its highest expression in monocytes implies that in human coronavirus infection, monocytes may also play a pivotal role in life-threatening exacerbations. Additionally, and surprisingly, IL6R basal expression in bronchial epithelia is very high. Respiratory endothelial cells have previously been identified as mediators of cytokine overexpression, which was ameliorated by sphingosine-1-phosphate $\left(\mathrm{S}_{1} \mathrm{P}_{1}\right)$ receptor agonism (Teijaro et al. 2011). The rescue of mice from hypercytokinaemia in this study may have been caused by the observed drastic reduction in circulating IL-6. 
104 Similarly, the elevated amounts of circulating activated sIL6R in human Covid-19 patients may be

105 reduced through scavenging by i.v. recombinant soluble gp130 protein (sgp130), thus reducing

106 cytokine storm risk or ameliorating its consequences. Importantly, this proposed scavenging

107 mechanism would interfere only with the deleterious trans-signalling of IL-6, keeping intact the

108 classic, pro-recovery cis-signalling (Garbers et al. 2015). This is an advantage over the IL6R-

109 antibody tocilizumab, which may without distinction interfere with any IL6R-mediated function

110 in all cells reached by the antibody; indeed, tocilizumab therapy conveys a high risk of severe

111 respiratory infection as its main adverse effect, even in RA basal therapy (Haraoui et al. 2017; Scott

112 2017). In addition, pathological studies revealed that tissues infected by SARS mainly comprised

113 airway epithelia, endothelia, monocytes, and macrophages (Gu et al. 2005). Thus, targeting of all

114 IL6R molecules regardless of cell type may worsen pathology by inhibiting the bodily response and

115 regenerative abilities.

116 A natural form of sgp130 is expressed via alternative splicing of the gp130 mRNA in normal

117 human blood (Müllberg et al. 1993). Human recombinant gp130 protein is readily available for

118 scientific purposes from a number of biotechnological suppliers, and a soluble gp 130-analog

119 coupled to the Fc portion of IgG (Olamkicept, Ferring Pharmaceuticals) is in Phase II clinical trial

120 against inflammatory bowel disease (IBD). Thus, production capacities for clinical investigation are

121 readily available. However, for an acute intervention in hypercytokinaemia (as opposed to chronic

122 treatment of RA or IBD), a non-modified bioidentical human recombinant sgp130 may be

123 preferable because of a lower immunogenicity. In these short-term scenarios, prolonged or

124 increased activity (as e.g. achieved by hybridisation to an antibody Fc portion) may not be needed. 
125 An open question is the timing of IL6R scavenging via i.v. sgp130. Since IL6R was elevated in

126 patients pre-exacerbation and the onset of cytokine storm as clinical phenomenon is rather rapid

127 and unpredictable (Tisoncik et al. 2012), there is need for a reliable biomarker of cytokine storm

128 onset. Several chemokines of the CCL and CXCL families may be sufficient markers because of

129 their significant up-regulation and accessibility via ELISA (Cheung et al. 2005). In spite of these

130 uncertainties, the expected toxicity of the bioidentical protein is low and thus, first-stage testing is

131 warranted. The details of clinical investigation of i.v. sgp130 in Covid-19 exacerbations will require

132 additional investigation into the kinetics and dynamics of cytokine storm, for instance via

133 longitudinal follow-up of patients with and without hypercytokinaemia, and adjustment of the

134 therapeutic algorithm in regard to other common treatment options such as antipyretics and

135 glucocorticoids (Bhaskar et al. 2020).

136

137 Declaration: no interests. 
140 Array data were downloaded as provided by the original authors from NCBI GEO, accession

141 number GSE5972. The original cohort (Cameron et al. 2007) included 50 SARS patients (22

142 males, 28 females) “without bias to age, sex, or previous medical history” from the Toronto area

143 and 10 healthy volunteers (five male, five female). Patients had a median age of 48 years (range 24-

144 83) and presented to the hospital at a median of two days after symptom onset (DSO). 57\% of non-

145 severe and $100 \%$ of severe cases received glucocorticoid treatment, with first dose given at a median

146 of six DSO. Given were either oral prednisone at a median peak daily dose of $100 \mathrm{mg}$, or

147 intravenous methylprednisolone at a median peak daily dose of $125 \mathrm{mg}$. For microarray analyses, 60

148 RNA samples (from peripheral blood) were collected from 40 patients at various time points

149 throughout the illness ( 1 sample from 30 patients and 2 to 5 samples from 10 patients). According

150 to time point, patients were stratified into three groups: pre-exacerbation, post-exacerbation, and

151 convalescent (exacerbation was determined by arterial oxygen saturation nadir); exacerbation

152 occurred at a median of eight DSO. Ten RNA samples (from peripheral blood) were collected

153 from the ten control subjects, adding up to 70 samples total. More detailed patient information can

154 be obtained from Table 1 of Cameron et al. (Cameron et al. 2007) and from subject metadata of

155 GSE5972.

156 Expression variance was analysed with R/quantro (Hicks \& Irizarry 2015) to guide the choice of

157 normalisation. Global differences were detected (via quantro()) in the four analysed groups $(\mathrm{p}=$

158 0.0198), and thus, no further quantile normalisation was applied. Permutation testing of the result

159 (1000 permutations) confirmed global differences with $\mathrm{p}<0.001$ (Supplementary Fig. 2). 
160 Differentially expressed genes were then sought using R/limma (Ritchie et al. 2015) using a model

161 of all four groups (control, pre-exacerbation, post-exacerbation, convalescent), controlling for sex

162 and age of subjects. Intensity ratio versus average intensity was assessed via $\operatorname{plot} M A()$, and

163 differential expression was assessed via the topTable() function, utilising Benjamini-Hochberg

164 correction of $\mathrm{p}$-values for all groups versus controls.

165 All code is available at https://github.com/slobentanzer/il6r-scavenging-by-sgp130.

166 Tissue-specific gene expression

167 For basal expression of interleukin-related genes in immune and control tissues, the "tissue-specific

168 regulatory circuits" of Marbach et al. (Marbach et al. 2016) were used. Briefly, for each tissue

169 regulatory circuit, transcriptional activity towards each gene was aggregated across all individual

170 transcriptional activators, i.e., the sum of weights of incoming edges of each gene of interest; the

171 authors have shown that this cumulative activity correlates with tissue-specific expression of each

172 gene (Marbach et al. 2016). Aggregated values were treated as expression matrix and further

173 analysed using $\mathrm{R}$, including visualisation via $\mathrm{R} /$ ggplot2 (Wickham 2016).

174 The code also is available at https://github.com/slobentanzer/il6r-scavenging-by-sgp130. 


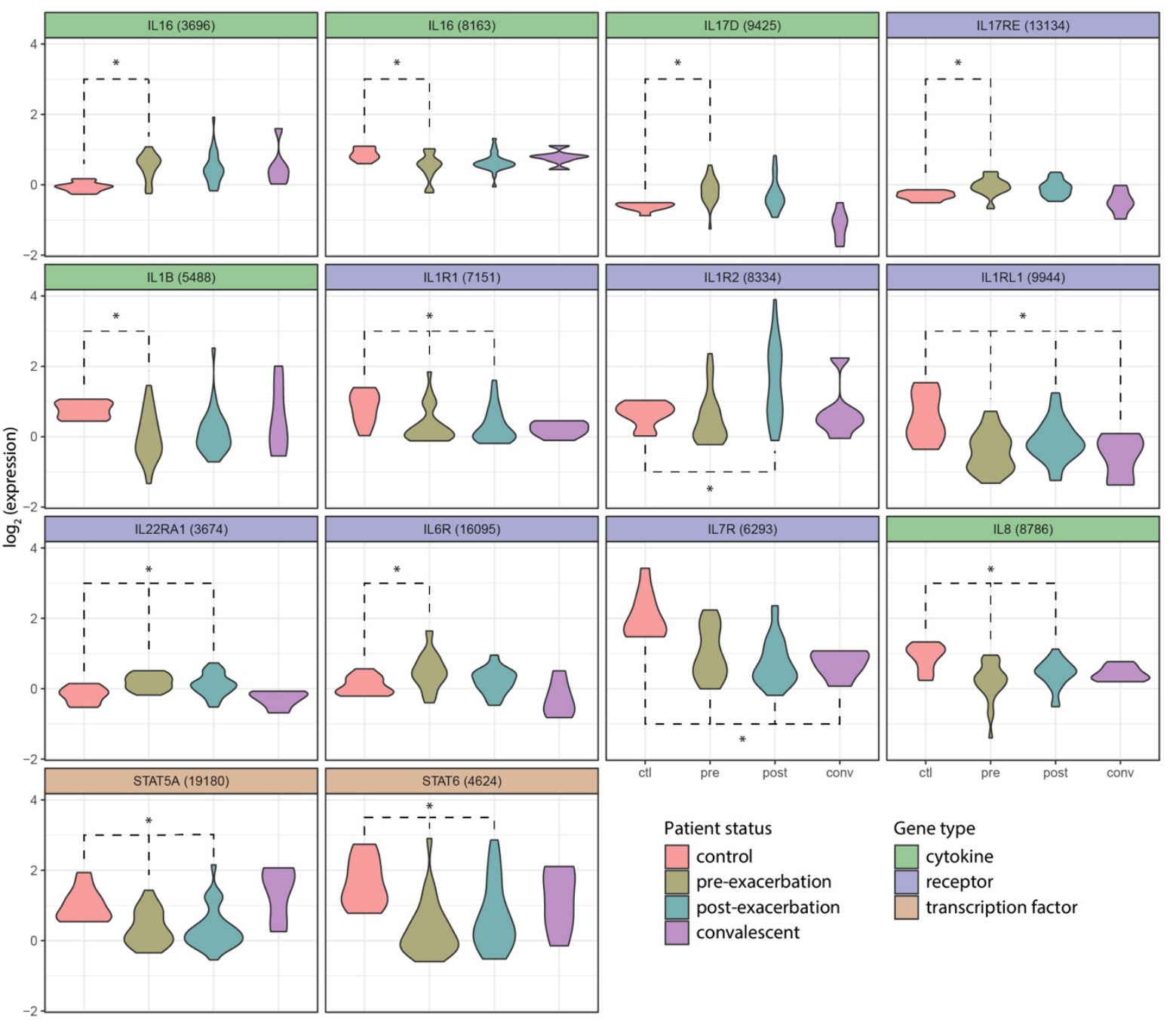

177 Figure 1: Differential expression of interleukin-related genes in a cohort of SARS (CoV 1) patients

178 from the Toronto area. Displayed are $\log _{2}$ fold expression values of interleukin (IL)-related

179 signalling molecules that exhibit differential expression (adjusted $\mathrm{p}$-value $<0.05$ ) in at least one

180 phase of the disease. The major pro-inflammatory cytokine IL-1 $\beta$ is decreased in patients pre-

181 exacerbation and slowly recovers until convalescence. The IL-1 $\beta$ receptors IL1R1 and IL1RL1 are

182 likewise decreased. In contrast, IL6R is elevated pre-exacerbation, and then returns to normal and

183 almost sub-normal levels during and after exacerbation. Downstream of IL receptor activation, 
184 STAT5A and STAT6 are decreased pre- and post-exacerbation, and return to normal levels upon

185 convalescence. ${ }^{*}: \mathrm{p}<0.05$.

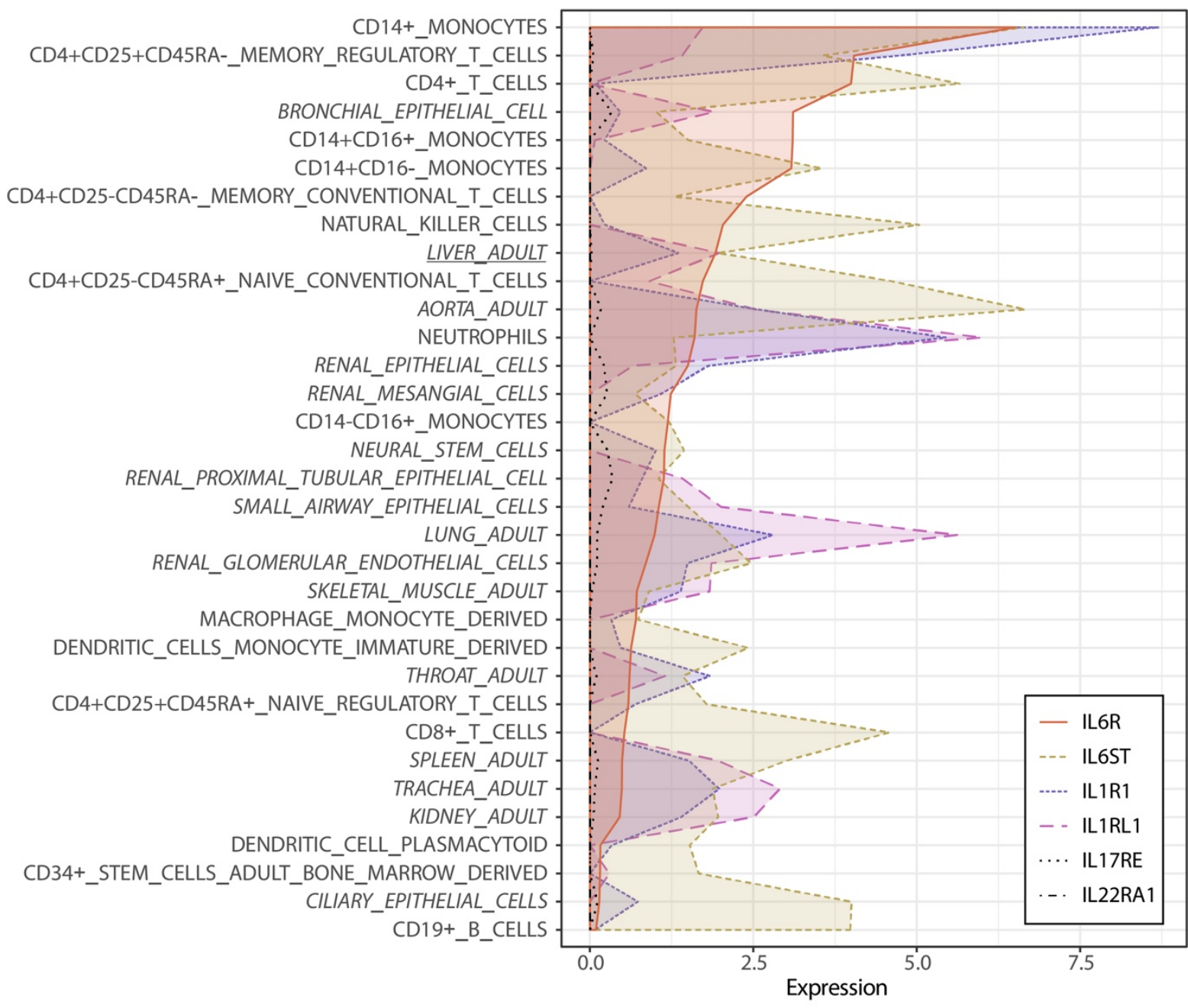

186

187 Supplementary Figure 1: Basal gene expression profiles of IL6R, gp130 (aka IL6ST), IL1R1,

188 IL1RL1, IL17RE, and IL22RA, as derived from "tissue-specific regulatory circuits" (Marbach et

189 al. 2016). As previously described, monocytes and regulatory T cells express highest amounts of

190 IL6R (solid line). Most non-immune control tissues, on the other hand, express very low levels of

191 IL6R (marked by italic font). However, bronchial epithelial cells express high amounts of IL6R,

192 even in comparison with hepatocytes, the only non-immune cells reported to show high mbIL6R 
194 particularly in non-immune tissues, whereas IL17RE and IL22RA1 show almost no basal

195 expression in the analysed tissues.

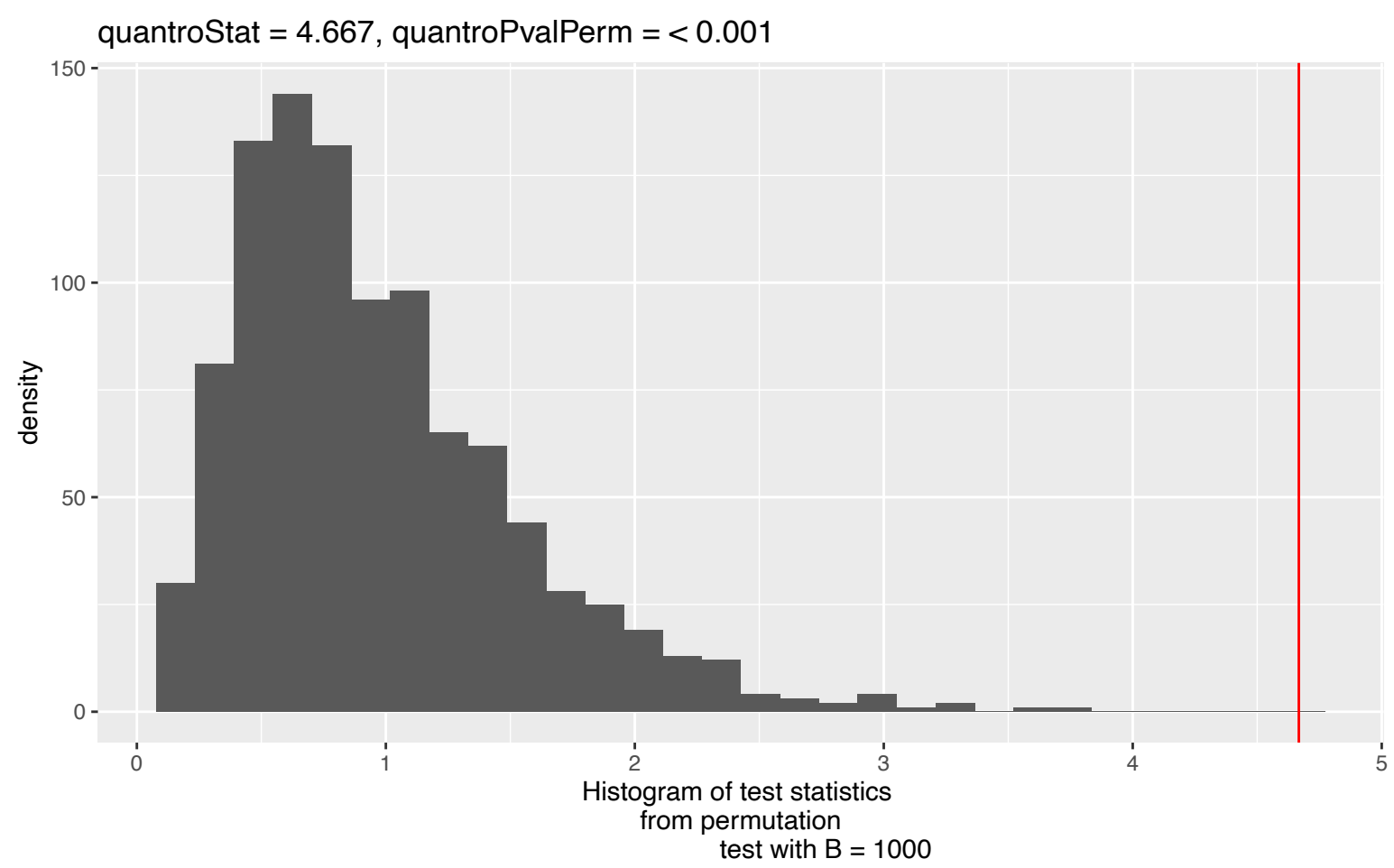

197 Supplementary Figure 2: Permutation test of quantro analysis of global differences in the 198 Toronto SARS cohort. 
200 Cytokine Storm In COVID-19 - Immunopathological Mechanisms, Clinical Considerations And 201 Therapeutic Approaches: The REPROGRAM Consortium Position Paper. Front. Immunol. doi: 10.3389/fimmu.2020.01648.

Cameron MJ, Ran L, Xu L, Danesh A, Bermejo-Martin JF, Cameron CM, Muller MP, Gold WL,

204 Richardson SE, Poutanen SM, et al. (2007). Interferon-Mediated Immunopathological Events Are Associated with Atypical Innate and Adaptive Immune Responses in Patients with Severe Acute Respiratory Syndrome. J. Virol. 81(16): 8692-8706. doi: 10.1128/JVI.00527-07. Guan Y, et al. (2005). Cytokine Responses in Severe Acute Respiratory Syndrome CoronavirusInfected Macrophages In Vitro: Possible Relevance to Pathogenesis. J. Virol. 79(12): 7819-7826. doi: 10.1128/JVI.79.12.7819-7826.2005.

211 Garbers C, Aparicio-Siegmund S \& Rose-John S (2015). The IL-6/gp130/STAT3 signaling axis:

212 Recent advances towards specific inhibition. Curr. Opin. Immunol. Elsevier Ltd. 34: 75-82. doi: $213 \quad 10.1016 /$ j.coi.2015.02.008.

214 Gu J, Gong E, Zhang B, Zheng J, Gao Z, Zhong Y, Zou W, Zhan J, Wang S, Xie Z, et al. (2005).

215 Multiple organ infection and the pathogenesis of SARS.J. Exp. Med. 202(3): 415-424. doi: $216 \quad 10.1084 /$ jem.20050828.

217 Haraoui B, Casado G, Czirják L, Taylor A, Bernasconi C, Reiss W \& Caporali R (2017). Patterns 218 of tocilizumab use, effectiveness and safety in patients with rheumatoid arthritis: core data results 219 from a set of multinational observational studies. Clin. Exp. Rheumatol. 35(6): 899-906. 
Hicks SC \& Irizarry RA (2015). quantro: a data-driven approach to guide the choice of an appropriate normalization method. Genome Biol. 16(1): 117. doi: 10.1186/s13059-015-0679-0. regulatory circuits reveal variable modular perturbations across complex diseases. Nat. Methods. 13(4): 366-370. doi: 10.1038/nmeth.3799. Rose-John S (1993). Differential shedding of the two subunits of the interleukin-6 receptor. FEBS Lett. 332(1-2): 174-178. doi: 10.1016/0014-5793(93)80507-Q.

Norelli M, Camisa B, Barbiera G, Falcone L, Purevdorj A, Genua M, Sanvito F, Ponzoni M, for cytokine-release syndrome and neurotoxicity due to CAR T cells. Nat. Med. 24(6): 739-748. doi: 10.1038/s41591-018-0036-4. differential expression analyses for RNA-sequencing and microarray studies. Nucleic Acids Res. 43(7): e47. doi: 10.1093/nar/gkv007. properties of the cytokine interleukin-6. Biochim. Biophys. Acta-Mol. Cell Res. 1813(5): 878-888. doi: 10.1016/j.bbamcr.2011.01.034. van Schie KA, Wolbink G-J \& Rispens T (2015). Cross-reactive and pre-existing antibodies to therapeutic antibodies-Effects on treatment and immunogenicity. MAbs. 7(4): 662-671. doi: 
241 Scott LJ (2017). Tocilizumab: A Review in Rheumatoid Arthritis. Drugs. 77(17): 1865-1879. doi:

$24210.1007 / s 40265-017-0829-7$.

243 Teijaro JR, Walsh KB, Cahalan S, Fremgen DM, Roberts E, Scott F, Martinborough E, Peach R,

244 Oldstone MBA \& Rosen H (2011). Endothelial Cells Are Central Orchestrators of Cytokine

245 Amplification during Influenza Virus Infection. Cell. 146(6): 980-991. doi:

$246 \quad 10.1016 /$ j.cell.2011.08.015.

247 Tisoncik JR, Korth MJ, Simmons CP, Farrar J, Martin TR \& Katze MG (2012). Into the Eye of

248 the Cytokine Storm. Microbiol. Mol. Biol. Rev. 76(1): 16-32. doi: 10.1128/MMBR.05015-11.

249 Wickham H (2016). ggplot2: Elegant Graphics for Data Analysis. New York: Springer-Verlag.

250

251 